\title{
Docking Studies and Evaluation of Anthranilic Acid Based PPAR $\delta$ Agonists for the Treatment of Metabolic Syndromes
}

\section{Meenu Beniwal ${ }^{1 *}$, Viney Lather ${ }^{2}$, Vikramjeet Judge ${ }^{3}$, Neelam Jain ${ }^{1}$ and Amit Beniwal ${ }^{4}$}

${ }^{1}$ Department of Pharmaceutical Education and Research, Bhagat Phool Singh Mahila Vishvavidyalaya, Khanpur Kalan, Sonepat, Haryana, India ${ }^{2}$ Department of Pharmaceutical Chemistry, JCDM College of Pharmacy, Sirsa, Haryana, India

${ }^{3}$ Department of Pharmaceutical Sciences, Guru Jambheshwar University of Science and Technology, Hisar, Haryana, India

${ }^{4}$ Combitic Global Caplet Pvt. Ltd., Sonipat, Haryana, India

\begin{abstract}
Metabolic syndrome (MS) is defined by a cluster of interconnected factors that directlyincrease the risk of coronary heart disease (CHD), other forms of cardiovascularatherosclerotic diseases (CVD) and Type 2 diabetes mellitus (T2DM). Several studies have suggested important role of PPARס in regulating lipid metabolism and energy homeostasis in muscle and fat. The present research work was undertaken to design and develop novel moieties derived from anthranilic acid scaffold as PPARס agonists that can be developed further for the management of metabolic disorders. The molecules were designed using receptor based drug design approach, by utilizing the X-ray crystallographic information of PPAR $\delta$ from PDB database. Based on the pharmacophoric requirements for PPAR $\delta$ binding, the anthranilic acid nucleus was chosen for the design of newer analogs by substitution of amide linker and introduction of lipophilic groups on aromatic system by using sulphonamide group as a linker. Amongst the several synthesized anthranilic acid derivatives, 5-chloro-2- [3-(4-nitro-phenylsulfamoyl)-benzoyl amino]-benzoic acid showed highest antidiabetic activity. The experimental results were found to be in concordance with that of the in silico results. Overall, this research work revealed the potential of novel anthranilic acid based PPARס agonists in the management of MS. Further, these molecules can serve as the starting point for the development of more potent lead molecules for MS.
\end{abstract}

Keywords: Anthranilic acid; PPAR $\delta$ receptor; Docking; Antidiabetic activity

\section{Introduction}

Metabolic syndrome (MS) is a complex disorder with high socioeconomic cost that is considered a worldwide epidemic. MS is defined by a cluster of interconnected factors that directly increase the risk of coronary heart disease (CHD), other forms of cardiovascular atherosclerotic diseases (CVD) and Type 2 diabetes mellitus (T2DM). Its main components are dyslipidemia (elevated triglycerides) and apolipoprotein B (apoB) containing lipoproteins, and low highdensity lipoproteins (HDL), elevation of arterial blood pressure (BP) and dysregulated glucose homeostasis, while abdominal obesity and/ or insulin resistance (IR) have gained increasing attention as the core manifestations of the syndrome [1]. The intense research interest in the PPARs, by the pharmaceutical industry and academia, stems largely from the well-documented therapeutic actions of their synthetic agonists in alleviating several of the maladies associated with MS [2].

PPARs (Peroxisome proliferator activated receptors) are ligandactivated transcription factors of the nuclear hormone receptor super family. They are activated by endogenous fatty acids and their metabolites. The three PPAR isoforms have been identified: PPAR $\alpha, \gamma$ and $\beta(\delta)$ which are differentially expressed in a tissue specific manner and play vital role not only in metabolic homeostasis but also in biological responses [3,4]. PPAR $\alpha$ receptor contains 468 amino acids and is highly expressed in tissues displaying enhanced capacities for fatty acid catabolism such as liver, heart, skeletal muscle, brown adipose tissue and kidney. PPAR $\gamma$ consists of 507 amino acids and is expressed as 2 subtypes, PPAR $\gamma 1$ and PPAR $\gamma 2$. The PPAR $\gamma 2$ subtype is distinct from PPAR $\gamma 1$ by the addition of 30 amino acids at $\mathrm{N}$-terminus [5]. PPAR $\gamma$ is almost exclusively expressed in adipose tissue. PPAR $\delta$ possesses 441 amino acids and is quite distinct from other PPARs by virtue of its ubiquitous tissue distribution [6].

The crystal structure of hPPAR $\delta$-LBD with GW0742 was determined at $1.95 \AA$ A resolution by Fernanda et al. (PDB ID: 3TKM) (Figure 1) [7].

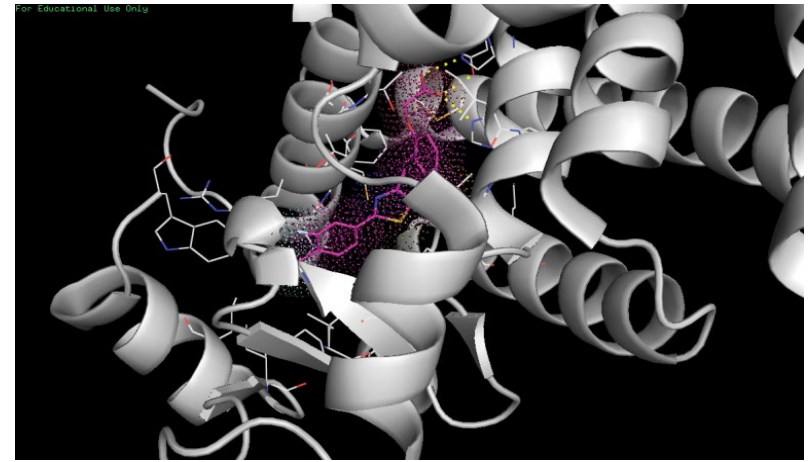

Figure 1: Crystallographic structure of the complex hPPARס-LBD: GW0742.

GW0742 occupied the Y-shaped ligand binding pocket and adopted a position predicted by the pharmacophoric model of PPAR ligands. The linker connecting the head and tail groups lies close to H3. In total, GW0742 made 29 ligand interactions with PPAR $\delta$ pocket, including three polar interactions and 26 apolar interactions [8].

Experimentally derived crystal and NMR protein structures are used as the basis for docking and the physics involved is based on what is known about atomic and molecular interactions as well as laws of thermodynamics. All docking methods basically include process

*Corresponding author: Meenu Beniwal, Department of Pharmaceutical Education and Research, Bhagat Phool Singh Mahila Vishvavidyalaya, Khanpur Kalan, Sonepat, Haryana-131 409, India, Tel: +919467878376; E-mail: hcpamit@gmail.com

Received December 21, 2015; Accepted January 20, 2016; Published January 25, 2016

Citation: Beniwal M, Lather V, Judge V, Jain N, Beniwal A (2016) Docking Studies and Evaluation of Anthranilic Acid Based PPARס Agonists for the Treatment of Metabolic Syndromes. Med chem 6: 023-032. doi:10.4172/2161-0444.1000318

Copyright: (c) 2016 Beniwal M, et al. This is an open-access article distributed under the terms of the Creative Commons Attribution License, which permits unrestricted use, distribution, and reproduction in any medium, provided the original author and source are credited. 
of sampling of ligand conformations, generating poses of the ligand within the receptor binding site, and scoring the poses [9].

\section{Experimental}

Scheme outlines the synthetic route followed for synthesis of anthranilic acid derivatives as reported in our recent work [10] (Scheme 1). The desired anthranilic acid derivatives were achieved through sulphonamides reactions with anthranilc acid and 5-chloro anthranilic acid as per reported scheme as follows:

\section{Docking studies}

To study the binding mode and explore the molecular interactions between the ligands and protein, docking studies were carried out for the designed anthranilic acid derivatives (1-33) with saluted cocrystallized structure of PPAR $\delta$ protein by using Glide 5.8 docking tool of Schrodinger Suit 2012 [9]. Following protocol was followed for the docking studies.

Ligand preparation: The 2D structures of the designed anthranilic acid derivatives were drawn by using Chem Sketch (ACDLABS 12.0) and stored in a database in sdf structure format [11]. All the structures were converted to $3 \mathrm{D}$ structure with the help of $3 \mathrm{D}$ optimization tool i.e., Ligprep of Schrodinger suit. By using the LigPrep 2.5 tool, the drawn ligands were geometry optimized by using the Optimized Potentials for Liquid Simulations-2005 (OPLS-2005) force field with the Steepest Descent method followed by the truncated Newton Conjugate gradient protocol. Partial atomic charges were computed using the OPLS-2005 force field. The LigPrep is a utility in Schrodinger software suite that combines tools for generating 3D structures from 1D (Smiles) and 2D (SDF) representation, searching for tautomers and steric isomers and geometry minimization of ligands. A maximum of 10 poses were generated for each ligand with different tautomeric and steric features for docking studies.

Protein preparation: The X-ray co-crystal structure of PPAR $\delta$ protein was obtained from the RCSB protein data bank (http:// www.rcsb.org/pdb). After evaluating the numbers of entries, the best protein (PDB entry 1YOS) was selected by analyzing all the proteins and chosing one with highest resolution i.e., 2.00 $\AA$. After selection, Protein preparation wizard of Schrodinger suite was used to prepare the protein. The selected PDB was preprocessed separately by deleting the substrate cofactor as well as the crystallographically observed water molecules (water without $\mathrm{H}$ bonds), correcting the errors in PDB file and optimizing the hydrogen bonds. After assigning charge and protonation state, finally energy minimization with root mean square deviation (RMSD) value of $0.30 \AA$ was done using OPLS2005 force field [11].

Validation of the docking protocol in glide: The most suitable
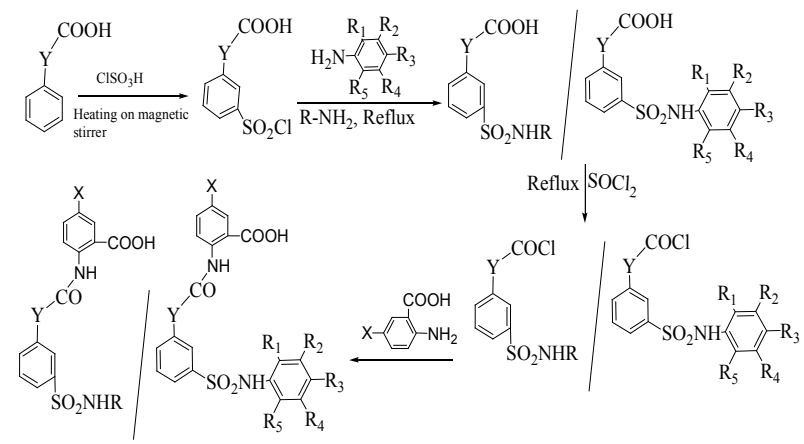

Scheme 1: [10] method of evaluating the accuracy of a docking procedure is to determine how closely the lowest energy pose predicted by the scoring function resembles an experimental binding mode as determined by $\mathrm{X}$-ray crystallography. In the present study, the docking of $1 \mathrm{Y} 0 \mathrm{~S}$ protein with its already presented ligand was performed to test the reliability and reproducibility of the docking protocol for our study. The root mean square deviation (RMSD) between the predicted conformation and the observed X-ray crystallographic conformation of the ligand by Glide ( $3 \AA$ ) was analyzed. This indicated the reliability of the docking protocol in reproducing the experimentally observed binding mode for target 1 YOS protein [11].

Generation of grid and ligand docking: Docking studies on designed derivatives prepared through Ligprep were carried out in the active site of the protein. Receptor Vander Waal's scaling for the non polar atoms was set to 0.9 which makes the protein site more spacious by moving back the surface of non polar regions of the protein and ligand. This kind of adjustment simulate to some extent the effect of breathing motion to the protein site, it is a kind of giving breathing to the receptor, this approach softens the active site region of the receptor making it flexible [12]. The prepared protein and the co-crystalized ligand were employed to build energy grids using the default value of protein atom scaling ( $1.0 \AA$ ) within a cubic box, centered on the centroid of the bound X-ray ligand. After Grid generation, the ligands were docked with the protein by using Glide 5.5 module (Glide, Version $5.8,2012$ ) in standard precision mode (SP) which uses MCSA (Monte Carlo Based Simulated Algorithm) based minimization. A total of 10 poses were generated for each of the ligand and the best docked pose (with lowest Glide Score value) obtained from Glide [13] was selected.

\section{Evaluation of antidiabetic activity}

Animals: Male Sprague Dawley (SD) rats (160-180 g) were procured from the disease free animal house of LLRVASV, Hisar. The animals were housed in standard polypropylene cages (three rats/cage) and maintained under controlled room temperature (22 \pm $\left.2^{\circ} \mathrm{C}\right)$ and humidity $(55 \pm 5 \%)$ with $12: 12 \mathrm{~h}$ light and dark cycle. All the rats were provided with commercially available rat normal pellet diet (NPD) (Ashirwad Feeds) and water ad libitum, prior to the dietary manipulation [14].

Induction of hyperglycaemia: A cohort of male Wistar rats was fasted overnight for at least $8 \mathrm{hr}$. Hyperglycaemia was induced in each fasted rat by administering alloxan monohydrate $(150 \mathrm{mg} / \mathrm{Kg}$ body weight; intraperitoneal) in normal saline. The control cohort was administered normal saline intraperitoneally. At $72 \mathrm{hr}$ post-induction of hyperglycaemia, blood glucose was assayed by the glucose oxidase method, using a glucometer (Accu-Chek Active, Roche Diagnostics). Only rats with established hyperglycaemia were included for subsequent treatment [14].

Treatment of hyperglycaemic rats with synthesized anthranilic acid derivatives: The selected anthranilic acid derivatives $(7,23,25$, 27 and 29) having dose of $30 \mathrm{mg} / \mathrm{kg}$ and tolbutamide $(20 \mathrm{mg} / \mathrm{kg})$ as standard were orally administered in $0.5 \%$ CMC (carboxy methyl cellulose) solution to a cohort of hyperglycaemic rats $(n=3)$. Untreated diabetic group received only the vehicle ( $0.5 \% \mathrm{CMC}$ solution).

Estimation of blood glucose: In the control, non-treated diabetic, and oral hyperglycaemic treated rats, blood glucose was assayed hourly $(0,2$ and $4 \mathrm{hr})$ by the glucose oxidase method [14] .

\section{Results and Discussion}

\section{Docking studies}

The docking simulations were performed by Glide 5.8, a docking tool 
of Schrodinger suit 2012, by energy minimization and optimization of designed anthranilic acid derivatives (1-33) in the active site of PPAR $\delta$ protein (PDB entry: 1YOS). For validating the docking protocol, the $1 Y 0 S$ protein was redocked with its co-crystallized ligand. The designed PPAR $\delta$ activators were located in the activator binding site, which is surrounded by helix- 3 and $\beta$-sheet, with the carboxylate directing away from the AF2 helix forming a hydrogen bond which is similar to the report of Shearer et al. [15] who have reported a similar arrangement for their synthesized anthranilic acid derivatives. All the 33 designed derivatives were docked in the active site. Out of these derivatives only $5,7,11,20,22,23,25,27,28,29$ showed appreciable binding in the binding site as determined by analysing the H-bond and hydrophobic interactions of the best docked poses. On the basis of Glide score and lowest Glide energy (Table 1), Comp. 7, 23, 27 and 29 were further analysed in detail by Molecular Visualization Tool, Pymol [16,17].

Binding pattern of synthesized anthranilic acid derivatives in the active site was compared with already reported active PPAR $\delta$ agonist GW9371 (PDB ID: 3DY6), in which only one H-bond interaction was observed with residue Thr288 (Bond distance: $2.8 \AA$ ). The binding pattern of GW9371 follows a different binding mode as compared to other PPAR $\delta$ agonist, where the acidic head group usually forms H-bonds with Tyr473, His323 and/or His449. Partial agonists however,

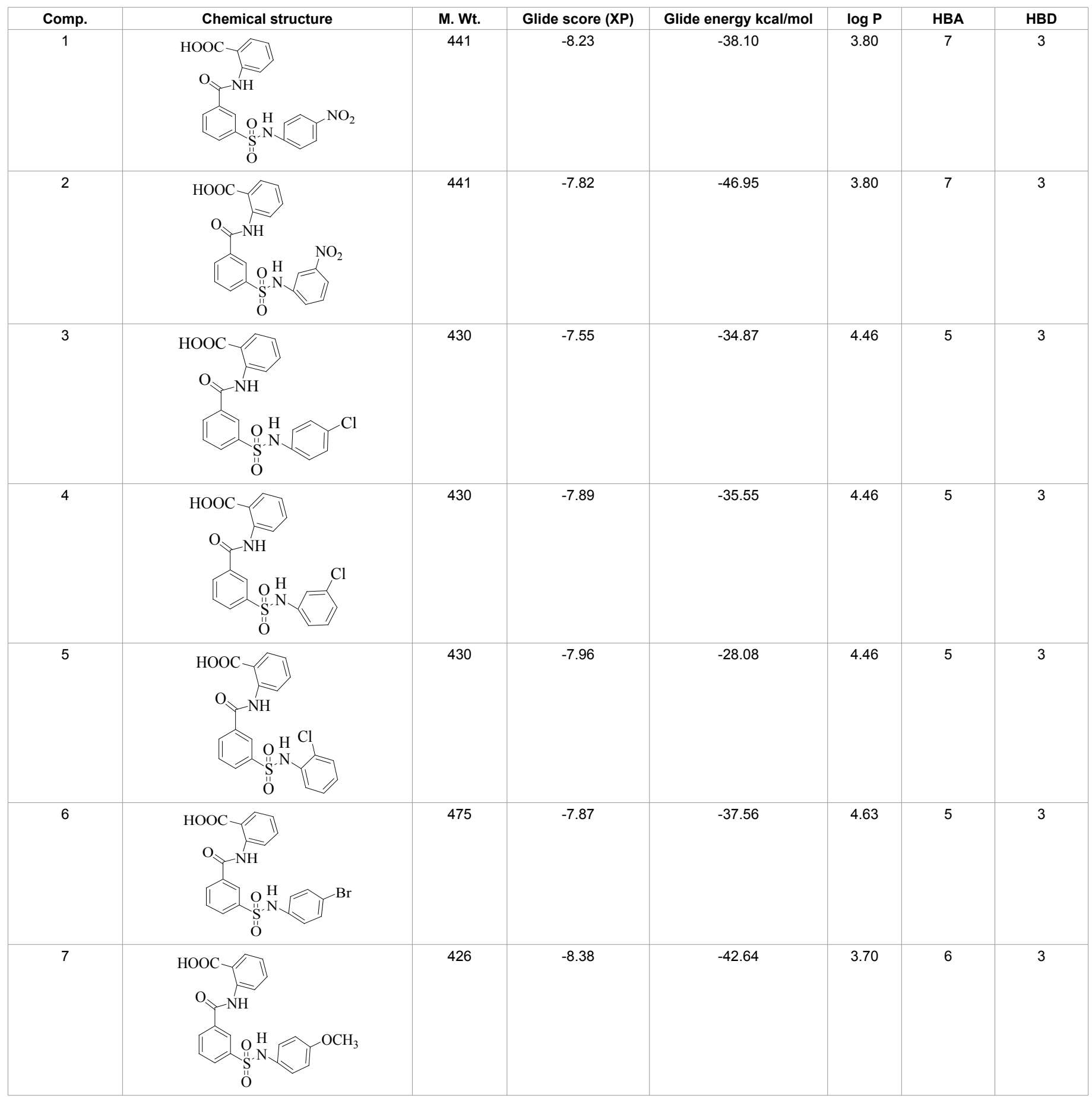


Citation: Beniwal M, Lather V, Judge V, Jain N, Beniwal A (2016) Docking Studies and Evaluation of Anthranilic Acid Based PPARס Agonists for the Treatment of Metabolic Syndromes. Med chem 6: 023-032. doi:10.4172/2161-0444.1000318

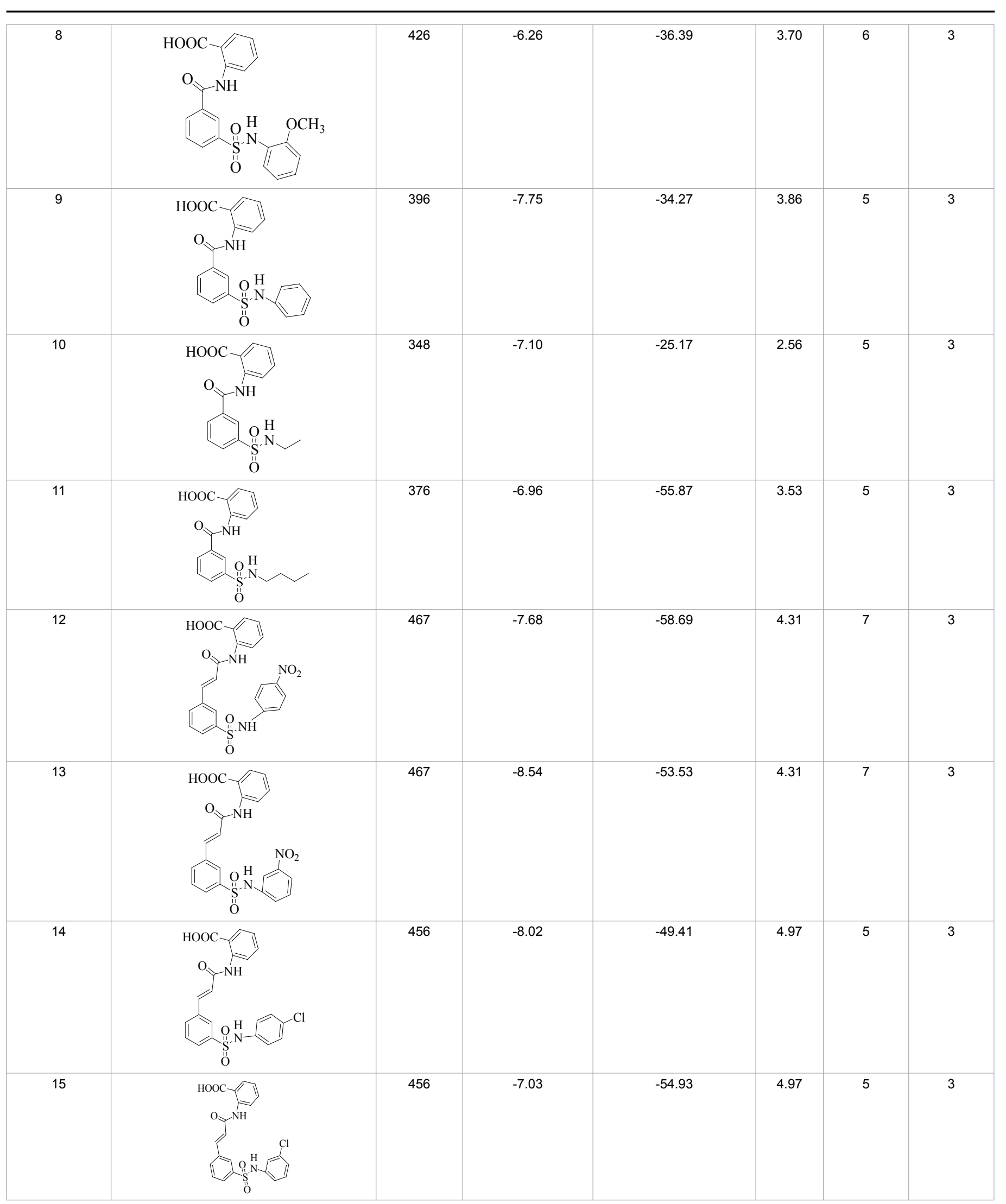


Citation: Beniwal M, Lather V, Judge V, Jain N, Beniwal A (2016) Docking Studies and Evaluation of Anthranilic Acid Based PPARס Agonists for the Treatment of Metabolic Syndromes. Med chem 6: 023-032. doi:10.4172/2161-0444.1000318

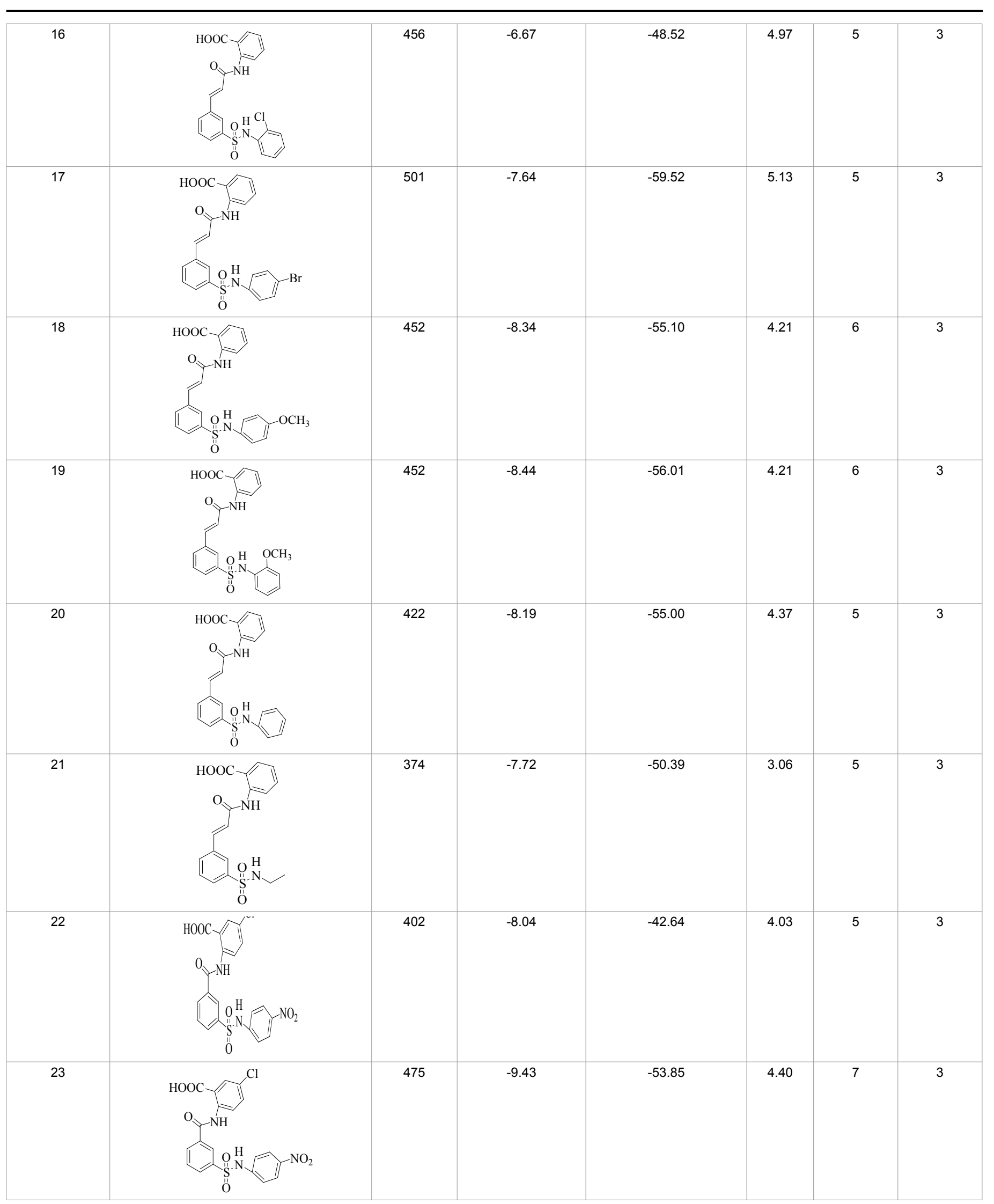


Citation: Beniwal M, Lather V, Judge V, Jain N, Beniwal A (2016) Docking Studies and Evaluation of Anthranilic Acid Based PPARס Agonists for the Treatment of Metabolic Syndromes. Med chem 6: 023-032. doi:10.4172/2161-0444.1000318

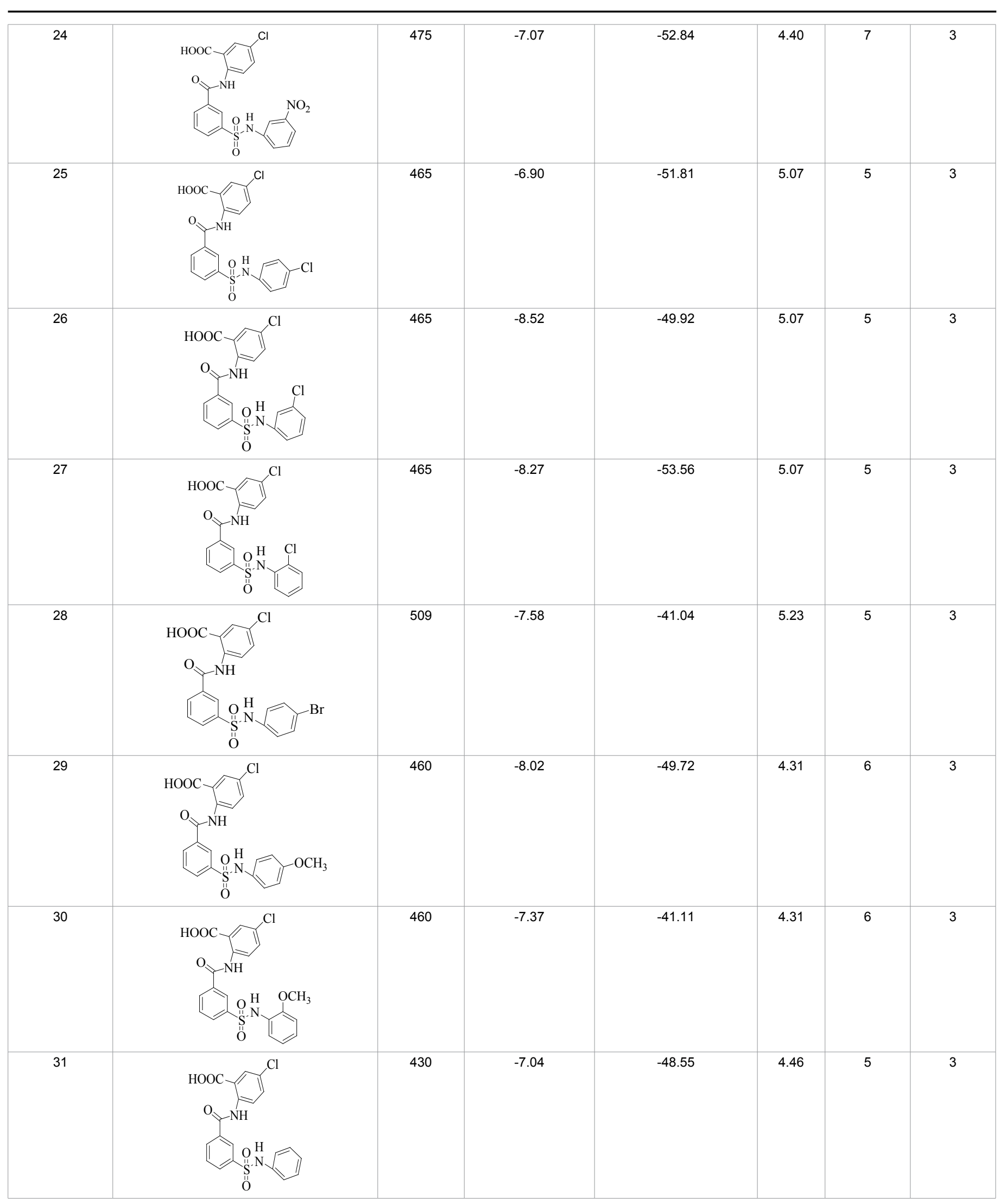




\begin{tabular}{|l|l|l|l|l|l|l|l|}
\hline 32 & 382 & -6.96 & 3.16 & 5 \\
\hline
\end{tabular}

log P, HBA, HBD, Mol. wt. were calculated by Marvin tools of Marvin Sketch v 5.5 [25]

Table 1: Glide score and molecular properties for synthesized anthranilic acid derivatives.

are also found to bind in a different manner as in case of GW9371. The docking results were found to be in accordance of the GW9371 ligand as shown by the overlay of docked poses of synthesized anthranilic acid derivatives on GW9371 and were studied to compare the binding interactions with the receptor site of PPAR $\delta$ as shown in Figures $2 b$, $3 \mathrm{~b}, 4 \mathrm{~b}$ and $5 \mathrm{~b}$.<smiles>O=C(Nc1ccccc1C(=O)O)c1cccc(S(=O)(=O)N2CCc3ccccc3C2)c1</smiles>

GW9371

In case of best docking pose of Comp.7, two H-bond interactions between the carboxylate group of anthranilic acid and Thr $288(2.72$ $\AA$ O-O distance) and Arg 284 (3.41 $\AA \mathrm{O}-\mathrm{N})$ were formed as shown in Figure 2a, which shows better binding interactions in comparison to GW9371, thus indicating that Comp. 7 could bind strongly to the PPAR $\delta$ receptor and can act as a better PPAR $\delta$ activator. Thr 288 is conserved in PPARa but not in PPAR $\gamma$, while Arg284 and Trp264 are unique to PPAR $\delta$. The sulphonamide adopted a conformation that directed the tail hydrophobic aryl/substituted aryl group towards Tyr473, His323, and His449. On visualizing the docked pose of Comp. 7 in the receptor site pockets, it was shown that the phenyl ring of $p$-methoxy group attains a specific position in one of the hydrophobic pockets on the right side showing interactions which provides an extra fitting in to the receptor site and thus improves its binding interactions.

The best docking poses of Comp. 23, 27 and 29 showed similar binding modes as that of Comp. 7 and formed H-bonds with Arg 284 (3.41 $\AA, 3.44 \AA$ and $2.88 \AA$ O-N distances respectively) and Thr288 (2.82 $\AA$, $2.57 \AA$ and $2.70 \AA$ O-O distances respectively) as shown in Figures $2 \mathrm{a}$, 3a, 4a and 5a. To verify the results, Comp. 23, 27 and 29 were overlaid with GW9371 ligand and showed similar type of binding mode. The sulphonamide also adopted a conformation as was in case of Comp.7 that directed the tail hydrophobic aryl/substituted aryl group towards Tyr473, His323, and His449. The results interpreted that differently substituted anthranilic acid derivatives showed a similar binding mode. The binding modes of chloro substituted compounds i.e., Comp. 23, 27,29 was found to be similar as that of Comp. 7 .

\section{In vivo antidiabetic activity}

CPCSEA guidelines emphasize on the minimization of animal

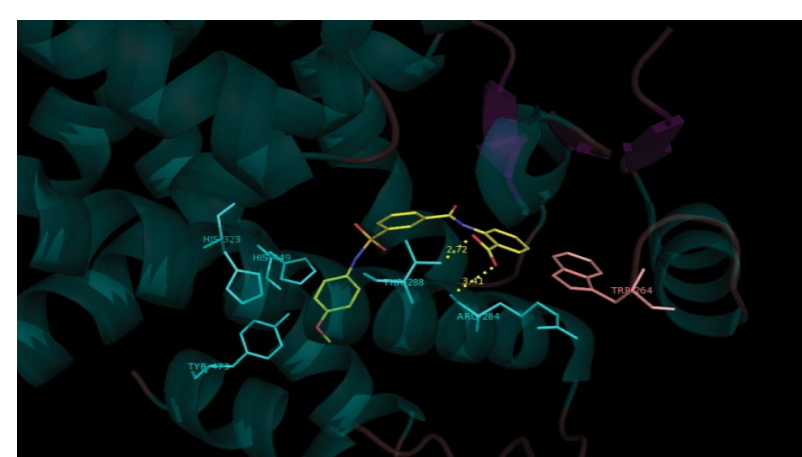

Figure 2a: Docked pose showing H-bond interaction for Comp. 7.

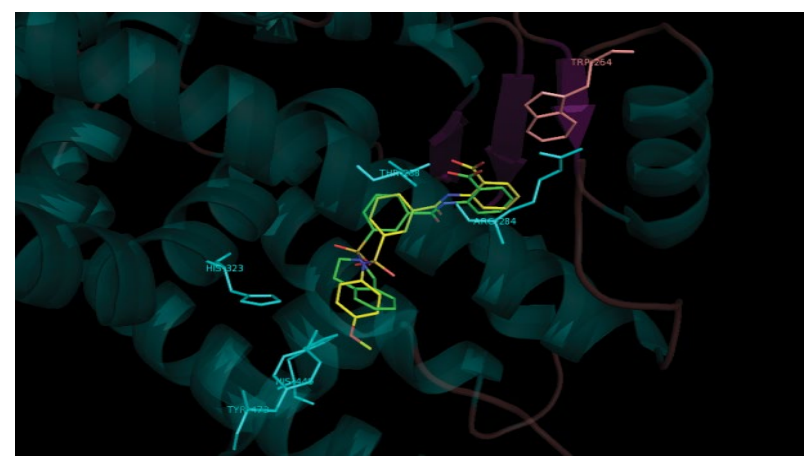

Figure 2b: Overlay of Comp. 7 (yellow) with GW9371 (green).

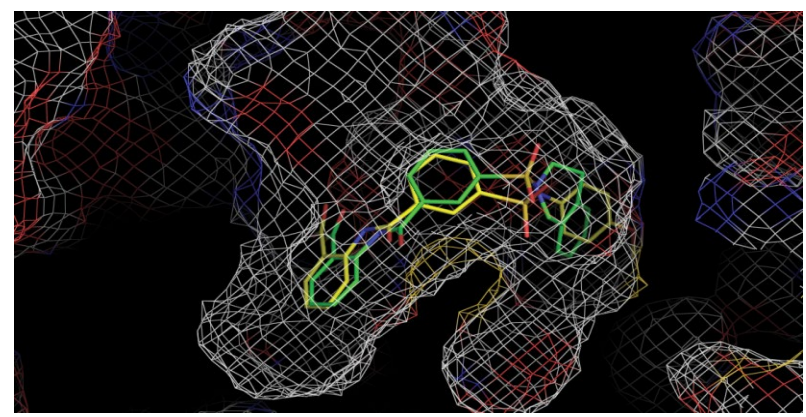

Figure 2c: Docked surface for Comp. 7 with GW9371. 
Citation: Beniwal M, Lather V, Judge V, Jain N, Beniwal A (2016) Docking Studies and Evaluation of Anthranilic Acid Based PPARס Agonists for the Treatment of Metabolic Syndromes. Med chem 6: 023-032. doi:10.4172/2161-0444.1000318

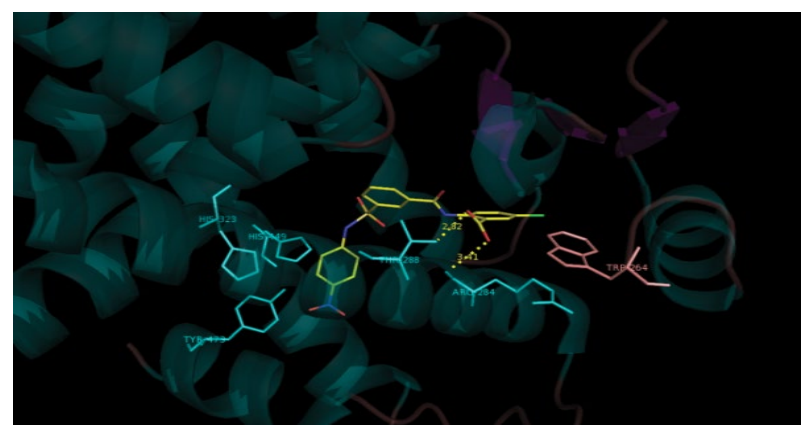

Figure 3a: Docked pose showing H-bond interaction for Comp. 23.

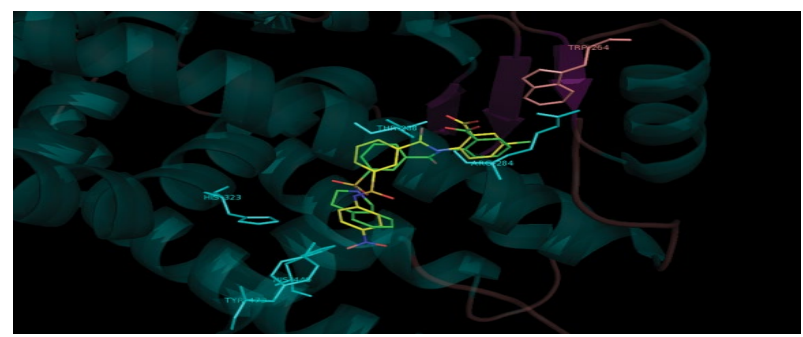

Figure 3b: Overlay of Comp. 23 (yellow) with GW9371 (green).

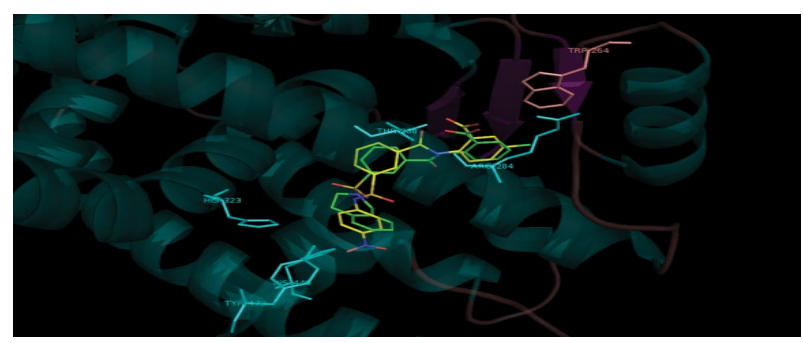

Figure 3c: Docked surface for Comp. 23 with GW9371.

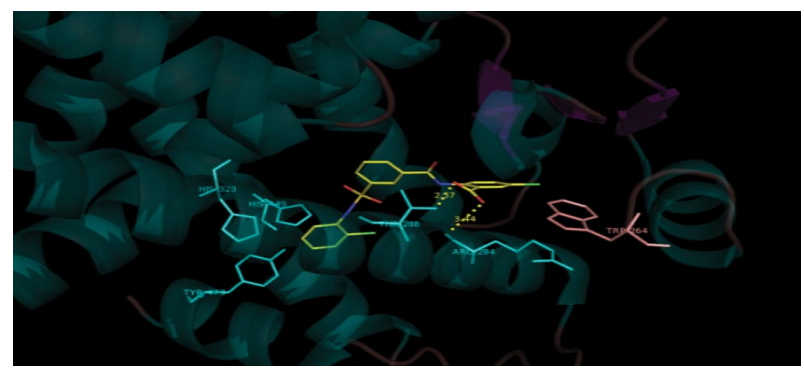

Figure 4a: Docked pose showing H-bond interaction for Comp. 27.

testing by using alternate screening methodologies like in vitro or in silico studies. Only selected molecules based on the docking studies were evaluated for the in vivo antidiabetic activity to reduce the number of test animals. The docking studies revealed that Comp.7, 23, 25, 27 and 29 had shown the similar binding mode as that of GW 9371 and strong interactions with PPAR $\delta$ protein and thus were selected further for in vivo antidiabetic activity. The results of antidiabetic activity measured as blood glucose levels at different time intervals are presented in Table 2 and Figure 6.

The in vivo antidiabetic studies revealed that Comp. 23, 25, 27 were found to be moderately active and amongst all Comp. 23 showed better potency. Comp. 7 and 29 were found to be ineffective in in vivo

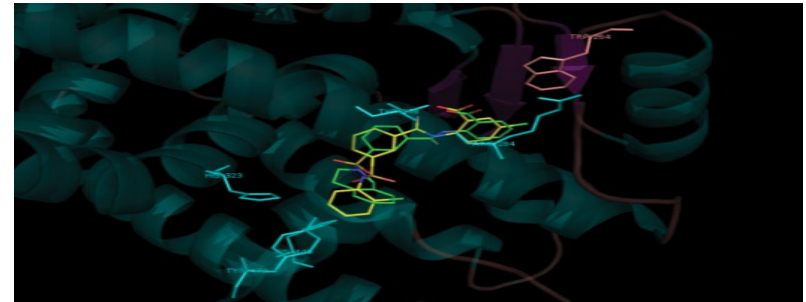

Figure 4b: Overlay of Comp. 27 (yellow) with GW9371 (green).

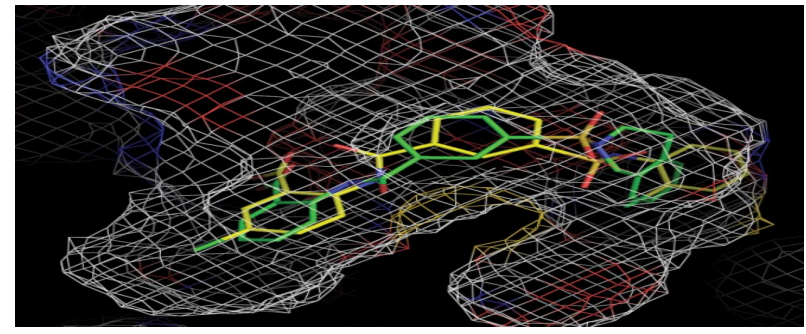

Figure 4c: Docked pose for Comp. 27 with GW9371.

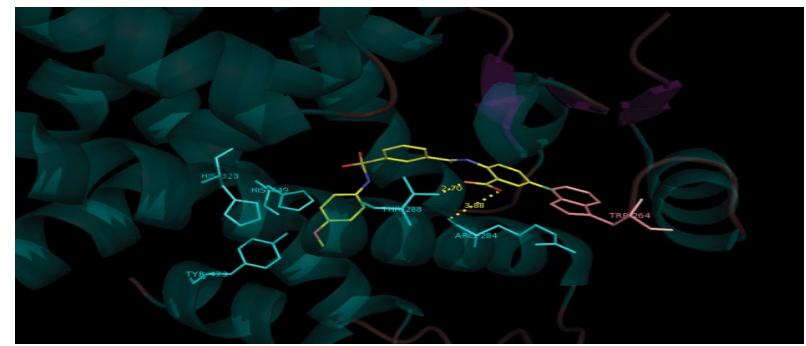

Figure 5a: Docked pose showing $\mathrm{H}$-bond interaction for Comp. 29.

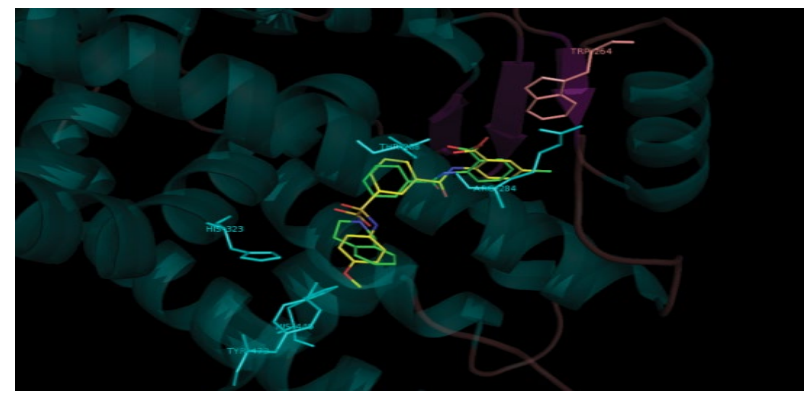

Figure 5b: Overlay of Comp. 29 (yellow) with GW9371 (green).

antidiabetic assay. The SAR for antidiabetic activity revealed the fact that compounds with $p$-nitro, $p$-chloro and $m$-chloro substituents on phenyl nucleus were found to be more effective in comparison to other synthesized anthranilic acid derivatives which may be attributed to the fact that these substituents fit excellently into the hydrophobic pockets and showed strong interaction with amino acids present there. These groups might have interacted strongly with a small hydrophobic region present at the extension of the hydrophobic pocket. The structures of Comp. 23, 25 and 27 can be further refined to get the lead compounds with potential activity against diabetes especially Comp. 23 i.e., 5-chloro-2-[3-(4-nitro-phenylsulfamoyl)-benzoylamino]-benzoic acid. 


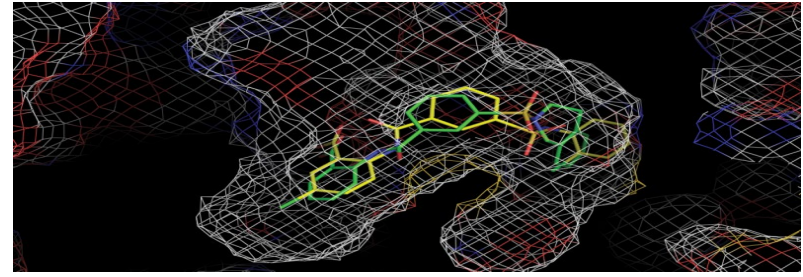

Figure 5c: Docked pose for Comp. 29 with GW9371

\begin{tabular}{|c|c|c|c|}
\hline \multirow{2}{*}{ Treatment } & \multicolumn{3}{|c|}{ Blood glucose level (mg/dL) } \\
\cline { 2 - 4 } & $\mathbf{0 ~ h r}$ & $\mathbf{2} \mathbf{~ h r}$ & $\mathbf{6} \mathbf{~ h r}$ \\
\hline Untreated & 272 & 272 & 274 \\
\hline Tolbutamide & 267 & 187 & 138 \\
\hline $\mathbf{7}$ & 302 & 295 & 290 \\
\hline $\mathbf{2 3}$ & $\mathbf{2 6 6}$ & $\mathbf{2 0 6}$ & $\mathbf{1 7 0}$ \\
\hline $\mathbf{2 5}$ & 298 & 248 & 220 \\
\hline $\mathbf{2 7}$ & 295 & 238 & 210 \\
\hline $\mathbf{2 9}$ & 235 & 228 & 220 \\
\hline
\end{tabular}

Values are mean of three measurements and measured in $\mathrm{mg} / \mathrm{dL}$

Table 2: Blood glucose level of selected molecules at different time intervals.

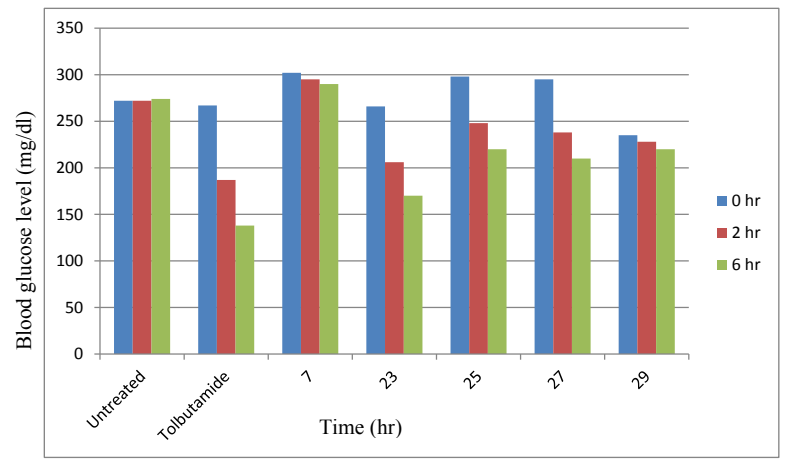

Figure 6: Blood glucose levels at different time intervals.

\section{Structure activity relationship}

From the results of antidiabetic activity, the following inference regarding structure activity relationship can be drawn:

1. The result of antidiabetic activity indicated that addition of electron withdrawing groups like $-\mathrm{NO}_{2}-\mathrm{Cl}$ increased the antidiabetic activity which can be seen from the antidiabetic activity results of Comp. 23, 25 and 27. These results are similar to the behaviour of electron withdrawing groups of acrylamides derivatives shown by Sidduri et al. [16].

2. Addition of electron donating group viz. $-\mathrm{OCH}_{3},-\mathrm{CH}_{3}$ etc. resulted in decrease in antidiabetic activity as seen in case of Comp. 7 and 29. The result of reduction in antidiabetic activity by introduction of electron donating group is supported by the study of Nikalje et al. acetamide derivatives [17].

\section{Conclusion}

The present research work was planned to design and develop novel moieties derived from anthranilic acid scaffold as PPAR $\delta$ agonists, which can be developed further for the management of metabolic disorders, the most threatening problem in the modern world. The molecules were designed using receptor based drug design approach, by utilizing the X-ray crystallographic information of PPAR $\delta$ from PDB database. Amongst the very few reported PDB entries, PDB entry:
1Y0S (Resolution: $2.00 \AA$ ) was selected for the rational drug design of new anthranilic acid derivatives. The study of binding sites of $1 Y 0 S$ and 3DY6 revealed the importance of various residues for $\mathrm{H}$-bond interactions i.e., Arg 284 and Thr 288 and other residues for the lipophilic interactions. Based on the pharmacophoric requirements for PPAR $\delta$ binding, the anthranilic acid nucleus was chosen for the design of newer analogs by substitution of amide linker and introduction of lipophilic groups on aromatic system by using sulphonamide group as a linker. Amongst the several synthesized anthranilic acid derivatives, Comp. 23 showed higher antidiabetic activity. The experimental results were found to be in concordance with that of the in silico results. The molecular properties of these new derivatives also followed the Lipinski rule of five, which predicts that for a molecule to have a drug like property, it should have Mol. Wt. $<500, \log \mathrm{P}<5, \operatorname{HBD}$ count $<5$, HBA count $<5$. Most of the synthesized molecules were found to have drug like properties as devised by Lipinski's rule of five. These new designed molecules can thus act as the starting point for the design and development of safe, effective and bioavailable PPAR $\delta$ agonists for the potential treatment of complications associated with MS. Overall, this research work reveals the potential of novel anthranilic acid based PPAR $\delta$ agonists in the management of MS and these molecules can serve as the starting point for development of more potent lead molecules for MS.

\section{References}

1. Akiyama TE, Meinke PT, Berger JP (2005) PPAR ligands: potential therapies for metabolic syndrome. Curr Diab Rep 5: 45-52.

2. Alberti KG, Zimmet $P$, Shaw J (2006) Metabolic syndrome- a new world-wide definition. A Consensus Statement from the International Diabetes Federation. Diabet Med 23: 469-480.

3. Ohashi M, Nakagone I, Kasuga JI, Nabusada H, Matsuno K, et al. (2012) Design synthesis and in vitro evaluation of a series of a-substituted phenylpropanoic acid PPAR? agonists to further investigate the stereochemistry- activity relationship. Bioorgan Med Chem 20: 6375-6383.

4. Willson TM, Brown PJ, Sternbach DD, Henke BR (2000) The PPARs: from orphan receptors to drug discovery. J Med Chem 43: 527-550.

5. Staels B, Fruchart JC (2005) Therapeutic role of peroxisome peroliferator activated receptor agonists. Curr Diab Rev 54: 2533-2540.

6. Guan Y (2004) Peroxisome proliferator-activated receptor family and its relationship to renal complications of the metabolic syndrome. J Am Soc Nephrol 15: 2801-2815.

7. Batista FAH, Daniela BB, Amanda B, Joyce G, Paulo SLO, et al. (2012) Structural insights into Human PPAR-delta selective ligand binding. PLoS ONE 7: e33643.

8. (2015) An Information Portal to 115031. Biological Macromolecular Structures A Structural View of Biology.

9. Glide (2013) Version 5.8, Schrödinger, LLC, New York, NY, USA.

10. Beniwal M, Jain N, Beniwal A (2015) Synthesis, characterization and biological evaluation of anthranilic acid derivatives. Eur J Biomed Pharm Sci 2: 1375 1392.

11. ChemSketch, Version 12.01, Advanced Chemistry Development, Inc., Toronto, ON, Canada.

12. Taverna DM, Goldstein RA (2002) Why are proteins marginally stable? Proteins 46: 105-109.

13. Friesner RA, Banks JL, Murphy RB, Halgren TA, Klicic JJ, et al. (2004) Glide: a new approach for rapid, accurate docking and scoring. 1. Method and assessment of docking accuracy. J Med Chem 47: 1739-1749.

14. Akinola O, Gabriel M, Suleiman A, Olorunsogbon F (2012) Treatment of alloxan-induced diabetic rats with metformin or glitazones is associated with amelioration of hyperglycaemia and neuroprotection. Open Diab J 5: 8-12.

15. Shearer BG, Patel HS, Billin AN, Way JM, Winegar DA, et al. (2008) Discovery of a novel class of PPARdelta partial agonists. Bioorg Med Chem Lett 18: 50185022 . 
Citation: Beniwal M, Lather V, Judge V, Jain N, Beniwal A (2016) Docking Studies and Evaluation of Anthranilic Acid Based PPARס Agonists for the Treatment of Metabolic Syndromes. Med chem 6: 023-032. doi:10.4172/2161-0444.1000318

16. Sidduri A, Grimsby JS, Corbett WL, Sarabu R, Grippo JF, et al. (2010) 2,3-Disubstituted acrylamides as potent glucokinase activators. Bioorg Med Chem Lett 20: 5673-5676.
17. Nikaljea APG, Choudharia S, Uneb H (2012) Design, synthesis and hypoglycemic activity of novel 2-(4-((2, 4- dioxothiazolidin-5-ylidene) methyl)-2methoxyphenoxy)-N- substituted acetamide derivatives. Eur J Ex Bio 2: 13021314. 